\title{
SOME PROPERTIES OF EIGENFUNCTIONS \\ OF LINEAR PENCILS AND APPLICATIONS TO MIXED TYPE OPERATOR-DIFFERENTIAL EQUATIONS
}

\author{
S. G. PYATKOV
}

Institute of Mathematics, Russian Academy of Sciences, Siberian Branch

Universitetskiน Prosp. 4, 630090 Novosibirsk, Russia

\begin{abstract}
In the first part of the paper we study some properties of eigenelements of linear selfadjoint pencils $L u=\lambda B u$. In the second part we apply these results to the investigation of some boundary value problems for mixed type second order operator-differential equations.
\end{abstract}

In this paper we consider linear pencils of the form

$$
L u=\lambda B u
$$

where $B, L$ are selfadjoint operators in a complex separable Hilbert space $E$. We suppose that $L$ is a positive operator, though almost the same results can be obtained in the case when the maximal nonpositive $L$-invariant subspace is finite-dimensional. Let $D(L) \subset E$ be the domain of $L$ and let $H_{1}$ be the completion of $D(L)$ with respect to the norm $\|u\|_{H_{1}}=(L u, u)^{1 / 2}$. We suppose that $D(L) \subset D(B)$ and that there are dense injections of $H_{1}$ in $E$ and $D\left(|B|^{1 / 2}\right)$. We define $F_{1}=\left(H_{1} \cap \text { ker } B\right)^{\perp}$ (the orthogonal complement in $\left.H_{1}\right)$ and we let $F_{0}$ be the completion of $F_{1}$ with respect to the norm $\|u\|_{F_{0}}=\left\||B|^{1 / 2} u\right\|_{E}$. The problem is whether or not the eigenfunctions of the problem (1) constitute a Riesz (unconditional) basis in $F_{0}$. The definition and some properties of Riesz bases can be found in [2]. This problem arises in consideration of boundary value problems for mixed type equations [9] and in the theory of selfadjoint operator pencils [11]. Apparently, the first papers devoted to this problem were $[6,1]$. We also mention the papers $[7,8,10]$.

The aim of the first part of this paper is to generalize some of the sufficient conditions from $[1,10]$. In the second part, we shall apply these results to some boundary value problems for mixed type second order operator-differential equa- 
tions. There are numerous papers devoted to such equations. In connection with our results we refer to $[3,4]$ where the existence and uniqueness of generalized solutions of a boundary value problem was proved. In this paper we shall prove the existence and uniqueness of solutions for two boundary value problems (the second is actually dual to the first). One of these problems was considered in $[3,4]$. We shall also investigate the question of the smoothness of solutions in dependence on that of the data. This problem is not so simple as in the case of hyperbolic or elliptic equations.

1. Preliminary results. Let $E^{+}, E^{-}, E^{0}$ be the spectral projectors of $B$ corresponding to the positive and negative parts of the spectrum of $B$ and to ker $B$. Thus if $U=E^{+}-E^{-}$then $U B=|B|=B U$. The operator $U$ is an isomorphism of $F_{0}$ onto $F_{0}$ and $U^{2}=I$. We define $F_{-1}$ to be the completion of $F_{0}$ with respect to the norm

$$
\|u\|_{F}=\left\|L^{-1} B u\right\|_{F}=\|B u\|_{H^{\prime}}
$$

where $H^{\prime}$ is the completion of $E$ with respect to the norm

$$
\|\varphi\|_{H^{\prime}}=\sup _{\psi \in H_{1}} \frac{|(\varphi, \psi)|}{\|\psi\|_{H_{1}}} .
$$

Throughout this paper $($,$) denotes the inner product in E$. An element $u \in F_{1}$ is an eigenfunction of the problem (1) if the equality (1) holds in $H^{\prime}$ for some $\lambda \neq 0$. Under our hypotheses the operator $L^{-1} B$ is bounded from $F_{1}$ to $F_{1}$ and we assume that it is completely continuous. Let $\left\{\varphi_{i}^{ \pm}\right\}$be the eigenfunctions of the problem (1) corresponding to the positive $\left(\lambda_{i}^{+}\right)$and negative $\left(\lambda_{i}^{-}\right)$eigenvalues. We assume that $\left\{\lambda_{i}^{+}\right\},\left\{-\lambda_{i}^{-}\right\}$are nondecreasing sequences.

Remark 1. We note that if $\operatorname{ker} B \cap H_{1}=\{0\}$ then $F_{1}$ coincides with $H_{1}$. Moreover, the case $F_{1} \neq H_{1}$ can be reduced to the case $F_{1}=H_{1}$. In fact, we take the orthogonal projector $P$ in $H_{1}$ on $F_{1}$ and define $B_{1}=B P+\left(I-P^{*}\right) B_{1}(I-P)$, where $B_{1}$ is any selfadjoint operator such that $H_{1} \subset D\left(\left|B_{1}\right|^{1 / 2}\right)$ and the condition $B_{1} v=0\left(v \in \operatorname{ker} B \cap H_{1}\right)$ implies that $v=0 ; P^{*}$ is the $E$-adjoint to $P$. Then $B_{1} v=B v$ for any $v \in F_{1}, F_{1}$ is an invariant subspace of $L^{-1} B_{1}: H_{1} \rightarrow H_{1}$ and ker $B \cap H_{1}=\{0\}$.

For convenience of the reader we now present some results from [10]. The next four theorems can be found there. Let $L(H, H)$ be the set of bounded linear operators from $H$ to $H$.

TheOREM 1. The eigenfunctions of the problem (1) normalized by $\left\|\varphi_{i}^{ \pm}\right\|_{F_{0}}=1$ constitute a Riesz basis in $F_{0}$ if and only if $\left[F_{1}, F_{-1}\right]_{1 / 2}=F_{0}$ (here $\left[F_{1}, F_{-1}\right]_{1 / 2}$ is defined by the complex interpolation method $[5,12])$.

THEOREM 2. Let $F_{s}=\left[F_{1}, F_{0}\right]_{1-s}$. Suppose that there exists $s>0$ such that $U \in L\left(F_{s}, F_{s}\right)$. Then $\left[F_{1}, F_{-1}\right]_{1 / 2}=F_{0}$. 
Let $M=E^{+} F, N=E^{-} F$.

Theorem 3. Suppose that in $F_{0}$ there exists a projector $P$ on $M$ or $N$ such that for some $s>0, P, P^{*} \in L\left(F_{s}, F_{s}\right) \cap L\left(F_{0}, F_{0}\right)$ ( $P^{*}$ is the $F_{0}$-adjoint $)$. Then there exists $s_{0}>0$ such that $U, E^{+}, E^{-} \in L\left(F_{s_{0}}, F_{s_{0}}\right)$.

Theorem 3 was formulated in [10] as Lemma 2.3 for $s=1$. It is easy to see that for $s<1$ the proof is the same.

In the sequel we assume that $\left(B \varphi_{i}^{ \pm}, \varphi_{j}^{ \pm}\right)= \pm \delta_{i j}$. We define

$$
P^{ \pm} f= \pm \sum\left(B f, \varphi_{i}^{ \pm}\right) \varphi_{i}^{ \pm} .
$$

If $\left[F_{1}, F_{-1}\right]_{1 / 2}=F_{0}$ then any $f \in F_{0}$ can be represented in the form $f=$ $\sum_{i=1}^{\infty}\left[\left(B f, \varphi_{i}^{+}\right) \varphi_{i}^{+}-\left(B f, \varphi_{i}^{-}\right) \varphi_{i}^{-}\right]=\left(P^{+}+P^{-}\right) f$. This series converges in $F_{0}$, and we can introduce an equivalent norm in $F_{0}$ by setting (see [10])

$$
\|f\|_{0}^{2}=\sum_{i=1}^{\infty}\left[\left|\left(B f, \varphi_{i}^{+}\right)\right|^{2}+\left|\left(B f, \varphi_{i}^{-}\right)^{2}\right|\right]
$$

Define $F_{0}^{ \pm}=\left\{u \in F_{0}: P^{ \pm} u=u\right\}$.

THEOREM 4. If $\left[F_{1}, F_{-1}\right]_{1 / 2}=F_{0}$ then there exist $P_{0}^{ \pm} \in L\left(F_{0}, F_{0}^{ \pm}\right)$such that

$$
E^{ \pm}\left(v-P_{0}^{ \pm} v\right)=0 \quad\left(\forall v \in F_{0}\right), \quad P_{0}^{ \pm} v=v, \quad P_{0}^{ \pm} E^{ \pm} v=v \quad\left(\forall v \in F_{0}^{ \pm}\right) .
$$

Now we shall give some new sufficient conditions which imply that $\left[F_{1}, F_{-1}\right]_{1 / 2}$ $=F_{0}$. They extend the conditions used in [1]. First we list some hypotheses.

1. There exists a space $\bar{H}_{1} \subset D\left(|B|^{1 / 2}\right)$ and linear operators $X \in L\left(H_{1}, \bar{H}_{1}\right)$, $Y \in L\left(\bar{H}_{1}, H_{1}\right)$ such that for some $c>0$

(a) $\left\|E^{+} \varphi\right\|_{F_{0}} \leq c\|X \varphi\|_{F_{0}}\left(\forall \varphi \in H_{1}\right)$

(b) $(|B| X \varphi, \psi)=(B \varphi, Y \psi)\left(\forall \varphi \in H_{1}, \forall \psi \in \bar{H}_{1}\right)$.

2. There exists a space $\bar{H}_{1} \subset D\left(|B|^{1 / 2}\right)$ and linear operators $X, Y \in L\left(\bar{H}_{1}, \bar{H}_{1}\right)$ such that

(a) $\left\|E^{+} \varphi\right\| \leq c\|X \varphi\|\left(\forall \varphi \in \bar{H}_{1}\right)$,

(b) $(|B| X \varphi, \psi)=(B \varphi, Y \psi)\left(\forall \varphi, \psi \in \bar{H}_{1}\right)$,

(c) if $\bar{F}_{1}=\left(\bar{H}_{1} \cap \operatorname{ker} B\right)^{\perp}$ then for some $s>0$

$$
\bar{F}_{s}=\left(\bar{F}_{1}, F_{0}\right)_{1-s}=\left(F_{1}, F_{0}\right)_{1-s} .
$$

3. There exist a space $\bar{H}_{1} \subset D\left(|B|^{1 / 2}\right), H_{1} \subset \bar{H}_{1}$ and linear operators $P, X, Y \in L\left(\bar{H}_{1}, \bar{H}_{1}\right)$ such that $P+Y X \in L\left(\bar{H}_{1}, H_{1}\right), \Delta P \subset \bar{H}_{1} \cap \operatorname{ker} B(\Delta P$ being the range of $P$ ) and

(a) $\left\|E^{+} \varphi\right\|_{F_{0}} \leq c\|X \varphi\|_{F_{0}}\left(\forall \varphi \in H_{1}\right)$,

(b) $(|B| X \varphi, \psi)=(B \varphi, Y \psi)\left(\forall \varphi, \psi \in \bar{H}_{1}\right)$.

TheOREM 5. Under any of these hypotheses $\left[F_{1}, F_{-1}\right]_{1 / 2}=F_{0}$. 
Proof. The proof in all cases is almost the same. For example, assume hypothesis 3 . We define

$$
W=\left\{u \in L_{2}\left(0, \infty ; F_{1}\right): d u / d t \in L_{2}\left(0, \infty ; F_{-1}\right)\right\} .
$$

Then $a \in\left[F_{1}, F_{-1}\right]_{1 / 2}$ if and only if there exists $u \in W$ such that $u(0)=a$ (see [5]) and we can set

$$
\|a\|_{\left[F_{1}, F_{-1}\right]_{1 / 2}}=\inf _{u(0)=a}\|u\|_{W}=\inf \left(\|u\|_{L_{2}\left(0, \infty ; F_{1}\right)}^{2}+\left\|\frac{d u}{d t}\right\|_{L_{2}\left(0, \infty ; F_{-1}\right)}^{2}\right)^{1 / 2} .
$$

Let us prove the injection $\left[F_{1}, F_{-1}\right]_{1 / 2} \subset F_{0}$. From this it already follows that $\left[F_{1}, F_{-1}\right]_{1 / 2}=F_{0}$ (see the proof of Theorem 2.2 in [10], or [1]). Let $a \in\left[F_{1}, F_{-1}\right]_{1 / 2}$. There exists $u \in W$ such that $u(0)=a$. There also exists a sequence $u_{n} \in C^{\infty}\left([0, \infty] ; F_{1}\right)[5]$ such that $\operatorname{supp} u_{n}$ is compact and $\left\|u_{n}-u\right\|_{W} \rightarrow 0$ as $n \rightarrow \infty$. Let $a_{n}=u_{n}(0) \in F_{1}$. We have (with $B^{+}=E^{+} B$ )

$$
\begin{aligned}
& \left(B^{+} a_{n}, a_{n}\right)=\left\|E^{+} a_{n}\right\|_{F_{0}}^{2} \leq c^{2}\left\|X u_{n}(0)\right\|^{2}=-2 c^{2} \int_{0}^{\infty} \operatorname{Re}\left(B X u_{n}^{\prime}(t), X u_{n}(t)\right) d t \\
= & -2 c^{2} \int_{0}^{\infty} \operatorname{Re}\left(B u_{n}^{\prime},(Y X+P) u_{n}\right) d t \leq c_{1}\left\|u_{n}^{\prime}\right\|_{F_{-1}}\left\|(Y X+P) u_{n}\right\|_{F_{1}} \leq c_{2}\left\|u_{n}\right\|_{W}^{2} .
\end{aligned}
$$

On the other hand,

$$
-\left(B a_{n}, a_{n}\right)=2 \int_{0}^{\infty} \operatorname{Re}\left(B u_{n}^{\prime}(t), u_{n}^{\prime}(t)\right) d t \leq c_{3}\left\|u_{n}\right\|_{W}^{2} .
$$

Using these inequalities we get

$$
\left(B^{-} a_{n}, a_{n}\right) \leq c_{4}\left\|u_{n}\right\|_{W}^{2}, \quad B^{-}=E^{-} B .
$$

Thus, we obtain

$$
\left\|a_{n}\right\|_{F_{0}}^{2} \leq\left(|B| a_{n}, a_{n}\right) \leq c\left\|u_{n}\right\|_{W}^{2} .
$$

Hence we deduce that $a=u(0) \in F_{0}$, i.e. $\left[F_{1}, F_{-1}\right]_{1 / 2}=F_{0}$.

Remark 2. We can use $E^{-}$instead of $E^{+}$in hypotheses 1-3. From now on, we assume that $\left[F_{1}, F_{-1}\right]_{1 / 2}=F_{0}$. Let $C([0,1] ; H)(H$ a Hilbert space) be the space of continuous functions from $[0,1]$ to $H$. Let

$$
\|\varphi\|_{s}^{2}=\sum_{n=1}^{\infty}\left(\left|\left(B \varphi, \varphi_{n}^{+}\right)\right|^{2}\left|\lambda_{n}^{+}\right|^{s}+\left|\left(B \varphi, \varphi_{n}^{-}\right)\right|^{2}\left|\lambda_{n}^{-}\right|^{s}\right) .
$$

If $s>0$ we set $F_{s}=\left\{v \in F_{0}:\|v\|_{s}<\infty\right\}$ and if $s<0$ we define $F_{s}$ as the completion of $F_{0}$ with respect to the norm \|\|$_{s}$. As already noted, the norms \|\|$_{F_{0}},\|\|_{0}$ are equivalent. We consider $B^{-1} L$ as an operator from $F_{0}$ to $F_{0}$. It is defined at least on $\operatorname{Lin}\left\{\varphi_{i}^{ \pm}\right\}$by means of the equality

$$
B^{-1} L\left(\sum\left(c_{i}^{+} \varphi_{i}^{+}+c_{i}^{-} \varphi_{i}^{-}\right)\right)=\sum\left(c_{i}^{+} \lambda_{i}^{+} \varphi_{i}^{+}+c_{i}^{-} \lambda_{i}^{-} \varphi_{i}^{-}\right) .
$$


It is easy to see that it is closable. So we can assume that $S=B^{-1} L$ is closed and in this case it is an isomorphism of $F_{2}$ and $F_{0}$. Thus, by induction we can introduce in $F_{s}(s$ an integer, $s>0)$ equivalent norms

$$
\|\varphi\|_{F_{k}}^{2}=\sum_{p=0}^{[k / 2]}\left\|S^{p} \varphi\right\|_{F_{k-2 p}}^{2} .
$$

Also, $S$ is an isomorphism of $F_{s}$ and $F_{s-2}$ with $S^{-1}=L^{-1} B$.

\section{Boundary value problems for mixed type operator-differential} equations. On the interval $(0,1)$ we consider the equation

$$
B u_{t t}-L u=B f
$$

with one of the following boundary conditions:

$$
\begin{aligned}
u(0) & =u_{0}, & E^{-}\left(u_{t}(0)-v_{0}\right) & =0, & E^{+}\left(u_{t}(1)-v_{1}\right) & =0, \\
u_{t}(0) & =v_{0}, & E^{-}\left(u(0)-u_{0}\right) & =0, & E^{+}\left(u(1)-u_{1}\right) & =0 .
\end{aligned}
$$

As mentioned above we assume that $\left[F_{1}, F_{-1}\right]_{1 / 2}=F_{0}$.

Theorem 6. Let $f \in C\left([0,1] ; F_{0}\right), u_{0} \in F_{1}, v_{0}, v_{1} \in F_{0}$. Then there exists a unique solution of the problem (2), (3) such that

$$
\frac{d^{k} u}{d t^{k}}=u^{(k)}(t) \in C\left([0,1] ; F_{1-k}\right), \quad k=0,1,2 .
$$

Pro of. We look for a solution of the problem (2), (3) in the form

$$
u=\sum_{k=1}^{\infty} u_{k}^{+}(t) \varphi_{k}^{+}+\sum_{k=1}^{\infty} u_{k}^{-}(t) \varphi_{k}^{-}=P^{+} u+P^{-} u .
$$

Then $u$ satisfies the boundary conditions

$$
P^{+} u(0)=u^{0}, \quad P^{+} u_{t}(1)=u^{1}, \quad P^{-} u(0)=v^{0}, \quad P^{-} u_{t}(0)=v^{1} .
$$

Each $u_{k}^{ \pm}$satisfies the equation

$$
u_{k t t}^{ \pm}-\lambda_{k}^{ \pm} u_{k}^{ \pm}=f_{k}^{ \pm}(t)= \pm\left(B f, \varphi_{k}^{ \pm}\right)
$$

and the boundary conditions

$$
\begin{array}{ll}
u_{k}^{+}(0)=u_{k}^{0}=\left(B u^{0}, \varphi_{k}^{+}\right), & u_{k t}^{+}(1)=u_{k}^{1}=\left(B u^{1}, \varphi_{k}^{+}\right), \\
u_{k}^{-}(0)=v_{k}^{0}=-\left(B v^{0}, \varphi_{k}^{-}\right), & u_{k t}^{-}(0)=-\left(B v^{1}, \varphi_{k}^{-}\right)=v_{k}^{1} .
\end{array}
$$

From (6), (7) we get

$$
\begin{aligned}
u_{k}^{+}(0)= & c_{1 k} \exp \left(\sqrt{\lambda_{k}^{+}}(t-1)\right)+c_{2 k} \exp \left(-\sqrt{\lambda_{k}^{+}} t\right) \\
& -\int_{0}^{t} \frac{\exp \left(-\sqrt{\lambda_{k}^{+}}(t-\xi)\right)}{2 \sqrt{\lambda_{k}^{+}}} f_{k}^{+}(\xi) d \xi-\int_{t}^{1} \frac{\exp \left(\sqrt{\lambda_{k}^{+}}(t-\xi)\right)}{2 \sqrt{\lambda_{k}^{+}}} f_{k}^{+}(\xi) d \xi,
\end{aligned}
$$


(9) $u_{k}^{-}(t)=v_{k}^{0} \cos \left(\sqrt{\left|\lambda_{k}^{-}\right|} t\right)+v_{k}^{1} \sin \left(\sqrt{\left|\lambda_{k}^{-}\right|} t\right)+\int_{0}^{t} \frac{\sin \left(\sqrt{\left.\left|\lambda_{k}^{-}\right|(t-\xi)\right)}\right.}{\sqrt{\left|\lambda_{k}^{-}\right|}} f_{k}^{-}(\xi) d \xi$.

$$
\begin{aligned}
& c_{1 k}=\frac{u_{k}^{0}}{2 \operatorname{ch}\left(\sqrt{\lambda_{k}^{+}}\right)}+\frac{u_{k}^{1} \exp \left(\sqrt{\lambda_{k}^{+}}\right)}{2 \sqrt{\lambda_{k}^{+}} \operatorname{ch}\left(\sqrt{\lambda_{k}^{+}}\right)}-\int_{0}^{1} \frac{\operatorname{sh}\left(\sqrt{\lambda_{k}^{+}} \xi\right)}{2 \sqrt{\lambda_{k}^{+}} \operatorname{ch}\left(\sqrt{\lambda_{k}^{+}}\right)} f_{k}^{+}(\xi) d \xi, \\
& c_{2 k}=\frac{u_{k}^{0} \exp \left(\sqrt{\lambda_{k}^{+}}\right)}{2 \operatorname{ch}\left(\sqrt{\lambda_{k}^{+}}\right)}-\frac{u_{k}^{1}}{2 \sqrt{\lambda_{k}^{+}} \operatorname{ch}\left(\sqrt{\lambda_{k}^{+}}\right)}+\int_{0}^{1} \frac{\operatorname{ch}\left(\sqrt{\lambda_{k}^{+}}(1-\xi)\right)}{2 \sqrt{\lambda_{k}^{+}} \operatorname{ch}\left(\sqrt{\lambda_{k}^{+}}\right)} f_{k}^{+}(\xi) d \xi,
\end{aligned}
$$

From (3), (8), (9) it follows that $u^{0}=P^{+} u_{0}, v^{0}=P^{-} u_{0}$, and

$$
E^{-}\left(v^{1}+B_{1} u^{1}\right)=E^{-}\left(v_{0}+g_{1}\right), \quad E^{+}\left(u^{1}+B_{2} v^{1}\right)=E^{+}\left(v_{1}+g_{2}\right),
$$

where

$$
\begin{gathered}
B_{1} u^{1}=\sum_{k=1}^{\infty} \varphi_{k}^{+} u_{k}^{1} / \operatorname{ch}\left(\sqrt{\lambda_{k}^{+}}\right), \quad B_{2} v^{1}=\sum_{k=1}^{\infty} \varphi_{k}^{-} v_{k}^{1} \cos \left(\sqrt{\left|\lambda_{k}^{-}\right|}\right), \\
\left(B g_{1}, \varphi_{k}^{-}\right)=0, \quad\left(B g_{1}, \varphi_{k}^{+}\right)=\frac{u_{k}^{0} \operatorname{sh}\left(\sqrt{\lambda_{k}^{+}}\right)}{\operatorname{ch}\left(\sqrt{\lambda_{k}^{+}}\right)}+\int_{0}^{1} \frac{\operatorname{ch}\left(\sqrt{\lambda_{k}^{+}}(\xi-1)\right)}{\operatorname{ch}\left(\sqrt{\lambda_{k}^{+}}\right)} f_{k}^{+}(\xi) d \xi \\
\left(B g_{2}, \varphi_{k}^{+}\right)=0, \quad\left(B g_{2}, \varphi_{k}^{-}\right)=v_{k}^{0} \sqrt{\left|\lambda_{k}^{-}\right|} \sin \left(\sqrt{\left|\lambda_{k}^{-}\right|}\right) \\
-\int_{0}^{1} \cos \left(\sqrt{\left|\lambda_{k}^{-}\right|}(1-\xi)\right) f_{k}^{-}(\xi) d \xi .
\end{gathered}
$$

By the definition of the norm in $F_{0}$ we find that $g_{1}, g_{2} \in F_{0}$.

We now show that there is at most one solution of the system (10) in $F_{0}$. There exist $\alpha, \beta \in F_{0}$ such that $E^{-} \alpha=0, E^{+} \beta=0$ and

$$
v^{1}+B_{1} u^{1}=\alpha, \quad u^{1}+B_{2} v^{1}=\beta .
$$

Then $v^{1}=P^{-} \alpha, u^{1}=P^{+} \beta$ and $P^{+} \alpha=B_{1} P^{+} \beta, P^{-} \beta=B_{2} P^{-} \alpha$. Hence

$$
\left\|P^{+} \alpha\right\|_{0}^{2}+\left\|P^{-} \beta\right\|_{0}^{2} \leq\left\|B_{1} P^{+} \beta\right\|_{0}^{2}+\left\|B_{2} P^{-} \alpha\right\|_{0}^{2} \leq\left\|P^{+} \beta\right\|_{0}^{2}+\left\|P^{-} \alpha\right\|_{0}^{2} .
$$

On the other hand, $(B \alpha, \alpha)=\left\|P^{+} \alpha\right\|_{0}^{2}+\left\|P^{-} \alpha\right\|_{0}^{2},-(B \beta, \beta)=\left\|P^{-} \beta\right\|_{0}^{2}-\left\|P^{+} \beta\right\|_{0}^{2}$. Thus from (11) it follows that $(B \alpha, \alpha)-(B \beta, \beta) \leq 0$, i.e. $\alpha=\beta=0$. From the second equation in (10) we get

$$
u^{1}+P_{0}^{+} E^{+} B_{2} v^{1}=P_{0}^{+} E^{+}\left(v_{1}+g_{2}\right) .
$$

The first equation in (10) yields

(12) $E^{-}\left(v^{1}-B_{1} P_{0}^{+} E^{+} B_{2} P_{0}^{-} E^{-} v^{1}\right)=E^{-}\left(v_{0}+g_{1}\right)-E^{-} B_{1} P_{0}^{+}\left(E^{+}\left(v_{1}+g_{2}\right)\right)$.

The second equation in (10) can be rewritten in the form

$$
E^{+} u^{1}+E^{+} B_{2} P_{0}^{-} E^{-} v^{1}=E^{+}\left(v_{1}+g_{2}\right) .
$$


So setting $\alpha=E^{-} v^{1}, \beta=E^{+} u^{1}$ we obtain an equivalent system

$$
\alpha-B^{-} \alpha=g^{-}, \quad \beta+B^{+} \alpha=g^{+} .
$$

By uniqueness of solutions for $(10), \operatorname{ker}\left(I-B^{-}\right)=\{0\}\left(B^{-}: F_{0} \rightarrow F_{0}\right)$. On the other hand, $\lambda_{i}^{+} \rightarrow \infty$ as $i \rightarrow \infty$ ( $L^{-1} B$ is completely continuous). This implies that $B_{1}$ and also $B^{-}$are completely continuous as operators from $F_{0}$ in $F_{0}$. Thus, a solution of systems (13) and (10) exists and $v^{1}, u^{1} \in F_{0}$. Using the representation (8), (9) of the solution $u(t)$ we can easily check that $u^{(k)}(t) \in C\left([0,1] ; F_{1-k}\right)$.

TheOREM 7. Let $f \in C\left([0,1] ; F_{-1}\right), v_{0} \in F_{-1}, u_{0}, u_{1} \in F_{0}$. Then there exists a unique solution of the problem $(2)$, (4) such that $u^{(k)} \in C\left([0,1] ; F_{-k}\right), k=0,1,2$.

The proof is analogous to that of Theorem 6 . We get here for $P^{-} u(0), P^{+} u(1)$ a system analogous to (10).

The question arises of the smoothness of the solutions in dependence on the smoothness of the data. Generally speaking, the smoothness of the solutions does not increase with the increase of the smoothness of the data. We need some orthogonality conditions if we want to say something about the smoothness of the solutions. These can be formulated in terms of the eigenfunctions of the problem (1) [8]. However, it seems simpler to formulate them in terms of some special solutions of the adjoint problem. We define

$$
\begin{aligned}
J_{3}\left(u_{0}, v_{0}, v_{1}, f, g\right)= & -\left(S^{k} u_{0}, S^{-1} g_{t}(1)\right)_{F_{1}}+\left(B S^{k} v_{1}, g(0)\right) \\
& -\left(B S^{k} v_{0}, g(1)\right)-\int_{0}^{1}\left(B S^{k} f(t), g(1-t)\right) d t, \\
J_{4}\left(v_{0}, u_{0}, u_{1}, f, g\right)= & \left(B S^{k} u_{1}, g_{t}(1)\right)-\left(B S^{k} u_{0}, g_{t}(0)\right) \\
& -\left(S^{k-1} v_{0}, g(1)\right)_{F_{1}}-\int_{0}^{1}\left(S^{k-1} f(t), g(1-t)\right)_{F_{1}} d t, \\
A_{k}=\left\{\left(g_{0}, g_{1}\right):\right. & \left.g_{0}, g_{1} \in D\left(S^{k}\right), E^{+} S^{k} g_{1}=0, E^{-} S^{k} g_{0}=0\right\} .
\end{aligned}
$$

TheOREM 8. Let $u_{0} \in F_{2 k+1}$ and $f \in C\left([0,1] ; F_{2 k}\right)(k=0,1,2, \ldots)$. Then there exists a solution $u$ of the problem $(2)$, (3) with $u^{(n)} \in C\left([0,1] ; F_{2 k+1-n}\right)$ $(n=0,1,2)$ if and only if for some $\bar{v}_{0}, \bar{v}_{1} \in F_{2 k}$ such that $E^{-}\left(\bar{v}_{0}-v_{0}\right)=0$, $E^{+}\left(\bar{v}_{1}-v_{0}\right)=0$ we have

$$
J_{3}\left(u_{0}, \bar{v}_{0}, \bar{v}_{1}, f, g\right)=0
$$

for any solution $g$ of the problem (2), (4) with data $v_{0}=0, f \equiv 0,\left(u_{0}, u_{1}\right) \in$ $A_{k}$. If such a solution exists and in addition $d^{n} f / d t^{n} \in C\left([0,1] ; F_{2 k+1-n}\right)(n=$ $1, \ldots, 2 k+1)$ then $u^{(n)} \in C\left([0,1] ; F_{2 k+1-n}\right)(n=1, \ldots, 2 k+1)$.

Remark 3. If $g_{i}(i=1,2)$ are the solutions of the problem (2), (4) with data $v_{0}^{i}=0, f^{i} \equiv 0, u_{0}^{i}, u_{1}^{i}$ and $E^{-}\left(u_{0}^{1}-u_{0}^{2}\right)=0, E^{+}\left(u_{1}^{1}-u_{1}^{2}\right)=0$ then $g_{1} \equiv g_{2}$. This is a consequence of Theorem 7 . 
Remark 4. Suppose that the operator $L$ has the property that if $E^{ \pm} g=0$ and $g \in D(S)$ then $E^{ \pm} S g=0$. In this case if (14) is valid for some $\bar{v}_{0}, \bar{v}_{1}$ then it holds for any $v_{0}, v_{1} \in D\left(S^{k}\right)$ such that $E^{-}\left(\bar{v}_{0}-v_{0}\right)=0, E^{+}\left(\bar{v}_{1}-v_{1}\right)=0$. This means that the orthogonality conditions (14) do not depend on $E^{+} v_{0}, E^{-} v_{1}$.

Pr o of. Suppose that (14) holds for some $\bar{v}_{0}, \bar{v}_{1}$. Let $v$ be a solution of the problem $(2),(3)$ with $v(0)=\bar{u}_{0}=S^{k} u_{0}, E^{-}\left(v_{t}(0)-S^{k} \bar{v}_{0}\right)=0, E^{+}\left(v_{t}(1)-S^{k} \bar{v}_{1}\right)=0$, $\bar{f}=S^{k} f$. We set $u=S^{-k} v$. Then $u^{(n)} \in C\left([0,1] ; F_{2 k+1-n}\right)(n=0,1,2)$. Let

$$
u_{j}(t)=\sum_{k=1}^{j}\left[\left(B u(t), \varphi_{k}^{+}\right) \varphi_{k}^{+}-\left(B u(t), \varphi_{k}^{-}\right) \varphi_{k}^{-}\right] .
$$

Then $u_{j} \in C^{2}\left([0,1] ; F_{2 k+2}\right)$ and

$$
\left\|u_{j}^{(n)}-u^{(n)}\right\|_{C\left([0,1] ; F_{2 k+1-n}\right)}+\left\|S^{k}\left(f-f_{j}\right)\right\|_{C\left([0,1] ; F_{0}\right)} \rightarrow 0 \quad \text { as } j \rightarrow \infty,
$$

where $f_{j}=u_{j t t}-S u_{j}$. Integrating by parts in

$$
\int_{0}^{1}\left(B\left(S^{k} u_{j t t}-S^{k+1} u_{j}\right), g(1-t)\right) d t=\int_{0}^{1}\left(S^{k} f_{j}, g(1-t)\right) d t
$$

where $g$ is a solution of the problem $(2),(4)$ with the data indicated earlier, we get

$$
J_{3}\left(u_{0 j}, \bar{v}_{0}, \bar{v}_{1}, f_{j}, g\right)+\left(B S^{k}\left(u_{j t}(1)-\bar{v}_{1}\right), g(0)\right)-\left(B S^{k}\left(u_{j t}(0)-\bar{v}_{0}\right), g(1)\right)=0
$$

$\left(u_{0 j}=u_{j}(0)\right)$. Letting $j \rightarrow \infty$ gives

(15) $J_{3}\left(u_{0}, \bar{v}_{0}, \bar{v}_{1}, f, g\right)+\left(B S^{k}\left(u_{t}(1)-\bar{v}_{1}\right), g(0)\right)-\left(B S^{k}\left(u_{t}(0)-\bar{v}_{0}\right), g(1)\right)=0$.

From $(14)$ and from $E^{+} S^{k}\left(u_{t}(1)-\bar{v}_{1}\right)=0, E^{-} S^{k}\left(u_{t}(0)-\bar{v}_{0}\right)=0$ it follows that

$$
\left(B\left(u_{t}(1)-\bar{v}_{1}\right), S^{k} g_{0}\right)-\left(B\left(u_{t}(0)-\bar{v}_{0}\right), S^{k} g_{1}\right)=0 .
$$

From the definition of $A_{k}$ and (16) we obtain $E^{+}\left(u_{t}(1)-v_{1}\right)=0, E^{-}\left(u_{t}(0)-v_{0}\right)=$ 0 . So $u$ is a smooth solution of the problem (2), (3).

Suppose now that $u$ is a solution of the problem (2), (3) of class mentioned in the theorem. Then repeating the previous arguments we get (15) where $v_{0}=u_{t}(0)$, $v_{1}=u_{t}(1)$ and hence (14) holds. The last assertion of the theorem follows directly from $(2)$.

Analogous arguments are used in the proof of the next theorem.

Theorem 9. Let $f \in C\left([0,1] ; F_{2 k-1}\right), v_{1} \in F_{2 k-1}$. Then a solution $u$ of the problem (2), (4) with $u^{(n)} \in C\left([0,1] ; F_{2 k-n}\right)(n=0,1,2)$ exists if and only if for some $\bar{u}_{0}, \bar{u}_{1} \in F_{2 k}$ such that $E^{-}\left(\bar{u}_{0}-u_{0}\right)=0, E^{+}\left(u_{1}-\bar{u}_{1}\right)=0$ we have

$$
J_{4}\left(v_{0}, \bar{u}_{0}, \bar{u}_{1}, f, g\right)=0
$$

for any solution $g$ of the problem (2), (3) with data $f \equiv 0, u_{0}=0, v_{0}=g_{0}$, $v_{1}=g_{1},\left(g_{0}, g_{1}\right) \in A_{k}$. If we have $(17)$ and in addition $f^{(n)} \in C\left([0,1] ; F_{2 k-n-2}\right)$ $(n=1, \ldots, 2 k)$ then $u^{(n)} \in C\left([0,1] ; F_{2 k-n}\right)(n=0,1, \ldots, 2 k+2)$. 
3. Examples. Here we do not give examples of application of Theorems 1-3 and 5 . These theorems are applicable to a wide class of differential operators $L$ if $B$ is the operator of multiplication by some function $g$ changing sign in the domain where $L$ is defined. Numerous examples can be found in $[1,7,8,10]$. Tricomi's equation is a well-known example. In the rectangle $D=[0,1] \times[-1,1]$ we consider the equation

$$
x u_{y y}+u_{x x}=f
$$

with boundary conditions

$$
\begin{array}{lll}
u(x, 0)=0 \quad(x \in(-1,1)), & u_{y}(x, 0)=v_{0}(x) \quad(x<0), \\
u_{y}(x, 1)=v_{1}(x) \quad(x>0), & u(-1, y)=u(1, y)=0 \quad(y \in(-1,1)) .
\end{array}
$$

We consider the boundary value problem (2), (3). Here $L=d^{2} / d x^{2}$ and $D(L)=$ $W_{2}^{2}(-1,1) \cap \stackrel{\circ}{W}_{2}^{1}(-1,1)$ (the definitions of the function spaces used here can be found in [12]). The space $E$ coincides with $L_{2}(-1,1), F_{1}=\stackrel{\circ}{W}_{2}^{1}(-1,1)$, and $F_{0}$ is the space of measurable functions with finite norm $\|u\|_{F_{0}}^{2}=\int_{-1}^{1}|x||u|^{2} d x$. Theorems $1-3$ and 5 are applicable here $[10,1]$ and the eigenfunctions of the problem (1) constitute a Riesz basis in $F_{0} . F_{k}$ is the set of all $u \in F_{0}$ with

$$
\int_{-1}^{1} \sum_{m=0}^{[k / 2]}\left|\left(\frac{1}{x} \frac{\partial^{2}}{\partial x^{2}}\right)^{m} u\right|^{2} d x+\left\|\left(\frac{1}{x} \frac{\partial^{2}}{\partial x^{2}}\right)^{[k / 2]} u\right\|_{F_{k-[k / 2]}}<\infty .
$$

In our case $A_{2 k}=\left\{\left(g_{0}, g_{1}\right) \in F_{2 k}:\left(\frac{1}{x} \frac{\partial^{2}}{\partial x^{2}}\right)^{k} g_{0}=0\right.$ almost everywhere on $(-1,0)$, $\left(\frac{1}{x} \frac{\partial^{2}}{\partial x^{2}}\right)^{k} g_{1}=0$ almost everywhere on $\left.(0,1)\right\}$. There are only $k$ functions $g_{0}$ linearly independent on $(-1,0)$ and $k$ functions $g_{1}$ linearly independent on $(0,1)$ such that $\left(g_{0}, g_{1}\right) \in A_{2 k}$. On these intervals $g_{0}, g_{1}$ are some polynomials. By Remarks 3,4 we can reformulate Theorem 8 as follows.

TheOREM 10. Let $f(x, y) \in C\left([0,1] ; F_{2 k+1}\right), u_{0} \in F_{2 k+1}$, and suppose $v_{0}, v_{1}$ coincide on $(-1,0),(0,1)$ respectively with some $\bar{v}_{0}, \bar{v}_{1} \in F_{2 k}$. Then a solution $u$ of the problem (18), (19) with

$$
\frac{\partial^{n}}{\partial y^{n}} u(x, y) \in C\left([0,1] ; F_{2 k+1-n}\right) \quad(n=0,1,2)
$$

exists if and only if the data of the problem satisfy the $2 k$ orthogonality conditions (here $\left.(f, g)=\int_{-1}^{1} f(x) \overline{g(x)} d x\right)$

$$
\begin{aligned}
& -\left(x\left(\frac{1}{x} \frac{\partial^{2}}{\partial x^{2}}\right)^{k} u_{0}, g_{t}(1)\right)+\left(x\left(\frac{1}{x} \frac{\partial^{2}}{\partial x^{2}}\right)^{k} \bar{v}_{1}, g(0)\right) \\
& \quad-\left(x\left(\frac{1}{x} \frac{\partial^{2}}{\partial x^{2}}\right)^{k} \bar{v}_{0}, g(1)\right)-\int_{0}^{1}\left(x\left(\frac{1}{x} \frac{\partial^{2}}{\partial x^{2}}\right)^{k} f(x, y), g(1-y, x)\right) d y=0
\end{aligned}
$$

for any solution $g(x, y)$ of the equation (18) with data $g_{y}(x, 0)=0, f=0$, $g(-1, y)=g(1, y)=0((x, y) \in D), g(0, x)=g_{0}(x)(x<0), g(1, x)=g_{1}(x)$ 
$(x>0)$, where $g_{0}, g_{1}$ belong to the above-mentioned function classes. If in addition $\partial^{n} f / \partial y^{n} \in C\left([0,1] ; F_{2 k-n+1}\right) \quad(n=1,2, \ldots, 2 k-1)$ then $\partial^{n} u / \partial y^{n} \in$ $C\left([0,1] ; F_{2 k-n+1}\right) \quad(n=1,2, \ldots, 2 k+1)$.

Remark 5. As shown by a number of examples, the spaces $F_{s}$ introduced here are most suitable for investigation of various boundary value problems where the spectral problem (1) arises.

\section{References}

[1] R. Beals, Indefinite Sturm-Liouville problems and half-range completeness, J. Differential Equations 56 (1985), 391-407.

[2] I. Ts. Gokhberg and M. G. Krein, Introduction to the Theory of Linear Nonselfadjoint Operators in Hilbert Space, Nauka, Moscow 1965 (in Russian); English transl.: Amer. Math. Soc., Providence, R.I., 1969.

[3] N. V. Kislov, Inhomogeneous boundary value problem for a second order operator-differential equation, Dokl. Akad. Nauk SSSR 280 (1985), 1055-1058 (in Russian); English transl. in Soviet Math. Dokl.

[4] _ - Boundary value problems for second order operator-differential equations of Tricomi type, Differentsial'nye Uravneniya 11 (1975), 718-720 (in Russian); English transl. in Differential Equations 11 (1975)

[5] J. L. Lions and E. Magenes, Non-Homogeneous Boundary Value Problems and Applications, Vol. 1, Springer, Berlin 1972.

[6] S. G. Pyatkov, Properties of eigenfunctions of a spectral problem and their applications, in: Well-Posed Boundary Value Problems for Nonclassical Equations of Mathematical Physics, Institute of Mathematics, Novosibirsk 1984, 115-130 (in Russian).

[7] —, On the solvability of a boundary value problem for a parabolic equation with changing time direction, Soviet Math. Dokl. 32 (1985), 895-897.

[8] - Properties of eigenfunctions of a spectral problem and their applications, in: Some Applications of Functional Analysis to Problems of Mathematical Physics, Institute of Mathematics, Novosibirsk 1986, 65-85 (in Russian).

[9] —, Solvability of boundary value problems for second order mixed type equations, in: Nonclassical Partial Differential Equations, Institute of Mathematics, Novosibirsk 1988, 77-90 (in Russian).

[10] - Some properties of eigenfunctions of linear sheaves, Siberian Math. J. 30 (1989), 587597.

[11] A. A. Shkalikov and V. T. Pliev, Compact perturbations of strongly damped pencils of operators, Mat. Zametki 45 (1989), 118-128 (in Russian).

[12] H. Triebel, Interpolation Theory, Function Spaces, Differential Operators, Deutscher Verlag Wiss., Berlin 1977, and North-Holland, Amsterdam 1978. 